\section{Kidney \\ Blood Pressure Research}

\title{
Identification of Key Genes and Pathways in Renal Cell Carcinoma Through Expression Profiling Data
}

\author{
Xiaoxia Liu Jinling Wang Guiling Sun \\ Department of Nephrology, Affiliated Hospital of Weifang Medical University, Weifang, Shandong, \\ China
}

\section{Key Words}

Renal cell carcinoma - Differentially expressed genes - Network - Functional enrichment • Pathways

\begin{abstract}
Background/Aims: To isolate key genes and pathways in renal cell carcinoma (RCC), which might reveal more evidences on the regulation network and contribute to pathogenic mechanisms of RCC. Methods: Microarray data of GSE34676, GSE23926 and GSE48008 were downloaded from Gene Expression Omnibus. Differentially expressed genes (DEGs) and differentially expressed miRNAs were respectively screened using Limma package, followed by the selection of CNV associated genes and miRNAs. A multi-molecular regulation interaction network was constructed, and significant modules were subsequently isolated from the network by Molecular Complex Detection (Mcode) of Cytoscape. Finally, GO terms and KEGG pathways of these genes and miRNAs in significant modules were enriched using DAVID. Results: Total 403 DEGs and 231 differentially expressed miRNAs were screened in RCC samples and normal group. Moreover, 1369 genes and 68 miRNAs were isolated by CNV analysis. Besides, a total of 59 miRNAs and 209 genes that related to 340 interaction pairs were analyzed and used to construct the network and 2 significant modules were identified. In the modules, CAV1 and CAV2 were shown to correlate with RCC. GNAI1, GPSM2 and GNAO1 were likely involved in the regulation of RCC through G protein signal transduction. Besides, G-protein coupled receptor protein signaling pathway, focal adhesion, MAPK signaling pathway and neuroactive ligand receptor interaction were enriched. Conclusion: Our study suggests that several crucial genes including CAV1,CAV2, GNAI1, GPSM2, and GNAO1 and pathways may play key roles in RCC progression.
\end{abstract}




\section{Kidney \\ Blood Pressure Research}

\section{Introduction}

Renal cell carcinoma (RCC) is one of the most common cancers in urinary system. The annual incidence of RCC represents 3\% of all malignancies in 2010 in the United States [1, 2]. Moreover, the incidence of RCC continues to be steadily rising over the past two decades [3]. In the treatment of RCC, it is still complicated due to different morphological characteristics and clinical phenotype, such as clear-cell RCC (82\%), type 1 or 2 papillary tumors $(11 \%)$, chromophobe tumors (5\%), and collecting duct carcinoma (1\%) [2, 4]. RCC is known to be resistant to all chemotherapeutic and radiation therapies, and surgical resection remains the mainstay of curative treatment to data [5]. However, studies have found that RCC partially responds to targeted therapies or immunotherapy [6, 7]. Exploring new therapeutic strategies for the treatment of this disease is imperative.

RCC is a disease with complex etiologies, which may be caused by the combined effect of multiple genes. Extensive efforts have been made to explore the underlying molecular pathogenesis of it. VHL (von Hippel-Lindau), p53, p16, p21 and p27 were shown as the main tumor suppressor genes in RCC, in which VHL and p53 were clearly certified to result in the development of RCC [8]. Dysfunction of VHL will lead to constitutively aberrant activation of the hypoxic response, like upregulation of vascular endothelial growth factor (VEGF), which is considered to play important roles in tumor development and angiogenesis [5, 9]. p53 is shown to suppress tumor growth and induce cell apoptosis in RCC [10, 11]. Moreover, miRNA-21 (miR-21) can post-transcriptionally downregulate the expression of programmed cell death 4 (PDCD4) and consequently promotes the proliferation and metastasis in RCC [12]. Besides, some factors involved in relevant biological pathways have been demonstrated as potential therapeutic targets, such as small-molecule multikinase inhibitors that target VEGF receptors (sunitinib and sorafenib) [13], the anti-VEGF antibody bevacizumab [14], and a mammalian target of rapamycin inhibitor temsirolimus [15]. Despite these great progresses in pathogenesis of RCC, the molecular mechanisms underlying this disease have not been fully elucidated.

Recently, microarray profiling of human RCC has increasingly been used to identify the potential genes and critical pathways involved in RCC [16]. In this study, expression profiles of gene, miRNA and SNP from RCC and normal group were respectively used to isolate differentially expressed genes (DEGs) and differentially expressed miRNAs. A multimolecular regulation interaction network that related to RCC was constructed and significant modules were subsequently identified. Moreover, functional enrichment analyses of these genes and miRNAs were performed. This study aimed to select several key genes and significant pathways that associated with RCC progression. These key genes and pathways might reveal some regulatory mechanisms and provide a new therapy approach in RCC.

\section{Materials and Methods}

\section{Microarray data and data preprocessing}

The gene expression profile of GSE23926 deposited by Gan et al. [17], miRNA expression profile of GSE48008 deposited by Zaravinos et al. [18], and SNP profile of GSE34676 deposited by Krill-Burger et al. [19] were downloaded from Gene Expression Omnibus (GEO, http://www.ncbi.nlm.nih.gov/geo/), which were based on the platforms of

GPL570: [HG-U133_Plus_2] Affymetrix Human Genome U133 Plus 2.0 Array,

GPL11434: miRCURY LNA microRNA Array, 6th generation - hsa, mmu \& rno, and

GPL6801: [GenomeWideSNP_6] Affymetrix Genome-Wide Human SNP 6.0 Array, respectively.

The information of patients in different samples from the three selected microarrays were shown in Table 1. The gene expression profile and miRNA expression profile was preprocessed using R language (v.2.13.0) [20]. CEL source files from all conditions were performed background correction and quartile data norma- 


\section{Kidney \\ Blood Pressure Research}

lization using RMA (Robust Multi-array Average) algorithm [21]. Midpoint of the probe set signal was considered as the expression levels of gene or miRNA if mutiple probe sets corresponded

Table 1. Information of patients used in the three selected microarrays

\begin{tabular}{lll}
\hline Microarray & Tumor samples & Control samples \\
\hline & Total $(\mathrm{n}=27)$ & Total $(\mathrm{n}=31)$ \\
& Chromophobe $(\mathrm{n}=5)$ & Normal renal $(\mathrm{n}=9)$ \\
GSE34676 & Clear cell $(\mathrm{n}=5)$ & Normal thyroid $(\mathrm{n}=14)$ \\
& Oncocytoma $(\mathrm{n}=5)$ & Normal lung specimens $(\mathrm{n}=8)$ \\
& Papillary type $1(\mathrm{n}=6)$ & \\
\hline & Papillary type 2 $(\mathrm{n}=6)$ & \\
\hline GSE23926 & with 100 nm 40HT treatment & without 100 nm 40HT treatment \\
& Total (n=31) & Total $(\mathrm{n}=19)$ \\
& With ccRCC $(\mathrm{n}=18)$ & \\
GSE48008 & With chRCC $(\mathrm{n}=3)$ & \\
& With papRCC $(\mathrm{n}=5)$ & \\
& With UUT-UCC $(\mathrm{n}=4)$ & \\
& Undifferentiated carcinoma $(\mathrm{n}=1)$ & \\
\hline
\end{tabular}
to the same

gene or miRNA. However, if a probe was mapped to multiple genes or miRNAs, the probe was considered unspecific and was then removed

In addition, APT (apt-probeset-genotype program in Affymetrix Power Tools) was used to analyze original CEL source files to obtain genotyping data and isolate SNP signal intensities of A, B allele [22]. Standard genotype clustering files were generated by genotyping data and signal intensities of normal samples. Finally, the signal intensities of A, B allele in cancer samples were consulted with standard genotype clustering files in order to evaluate Log R Ratio (LRR) and B Allele Frequency (BAF) of every locus in cancer samples.

Identification of DEGs and differentially expressed miRNAs

The T test in Limma package [23] was used to select the DEGs in RCC samples from GSE23926 and the differentially expressed miRNAs from GSE48008 compared with the normal samples. Fold change (FC) of the gene expression was also observed for differential expression test. The p-value $<0.05$ and $|\log 2 \mathrm{FC}|>1$ were considered as the threshold.

Copy number variation analysis

PennCNV [24] and Hidden Markov model (HMM) [25] were used to deduce the CNV (Copy number variation) regments in the genomes of microarray data GSE34676 based on the LRR and BAF of RCC samples. Then the occurrence of a same CNV sessions was counted in RCC samples. Sessions were retained which appeared in more than 7 samples. Finally, CNV sessions were annotated to select the genes or miRNAs related to $\mathrm{CNV}$.

\section{Prediction of miRNA target genes}

In order to improve the accuracy and reduce false positive, the target genes of differentally expressed miRNAs were predicted by 6 prediction algorithm, including PicTar [26], DIANA-microT [27], miRanda [28], RNAhybrid [29], RNA22 [30] and TargetScan [31]. The target genes which were appeared in at least three algorithms were identified as the truly target genes for the differentially expressed miRNAs.

\section{Network analysis and module mining}

The selected DEGs and differentially expressed miRNAs associated with CNV, and the target genes for miRNAs were used to construct the regulatory network of RCC using Mocde (Molecular Complex Detection) in Cytoscape [32]. Key biomoleculars were isolated in this network and significant modules with degree cutoff to 2 (degree $>2$ ) from the constructed network were analyzed.

Functional enrichment analysis of the genes in modules

Gene Ontology (GO) [33] and KEGG pathway [34] enrichment analysis for the selected DEGs in the modules were performed to explore significant biological processes and pathways using the DAVID (Database 


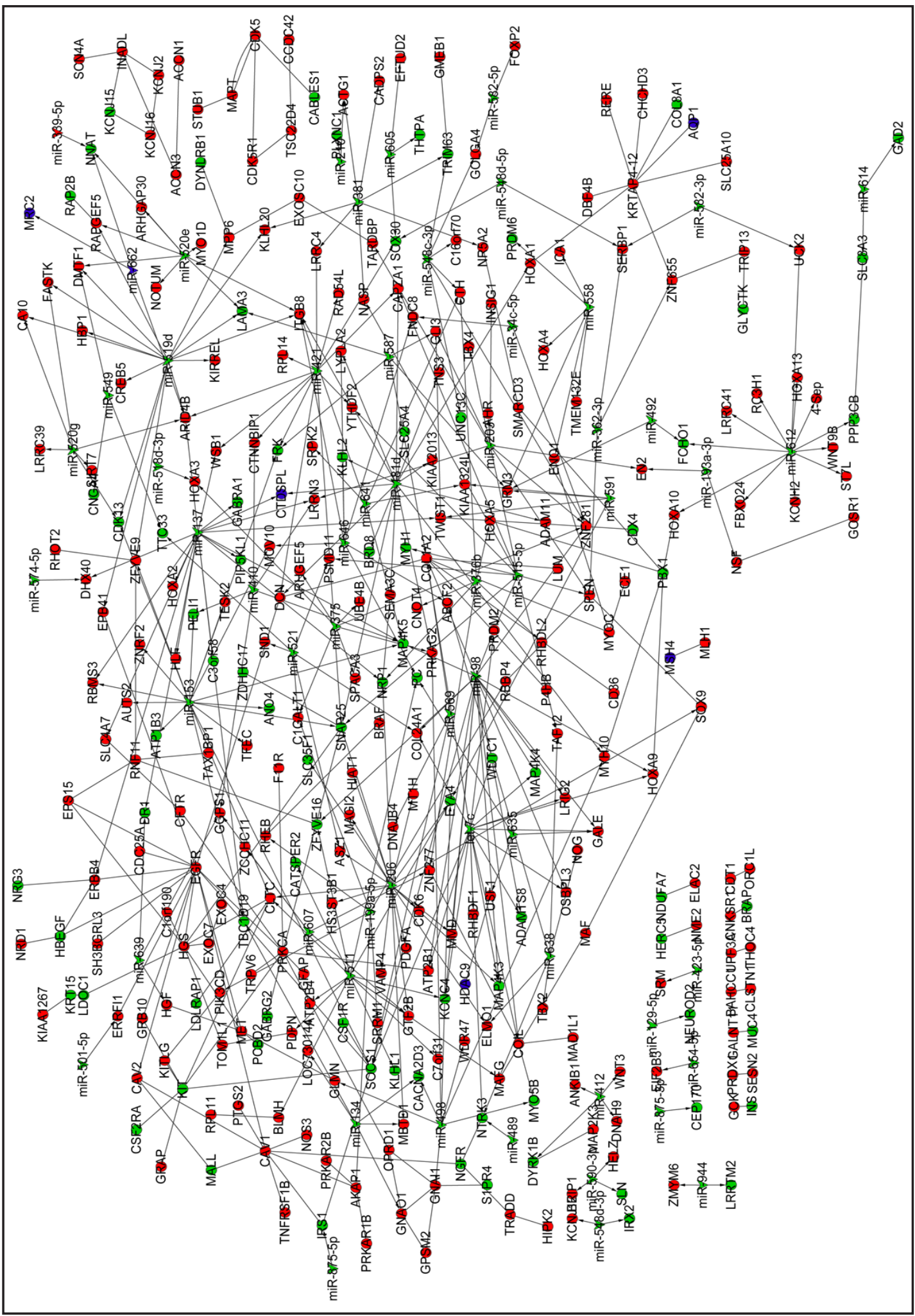

Fig. 1. Network constructed by regulation and protein-protein interactions. Circle represents related genes of RCC; triangle represents related miRNAs of RCC; green represents DEGs and miRNAs; red represent CNV related genes and miRNAs; blue represents both differentially expressed and CNV related genes and miRNAs. 


\section{Kidney \\ Blood Pressure Research}

Fig. 2. Two modules in specific RCC regulatory network. Green represents DEGs; red represents CNV related genes.

Table 2. GO functional enrichment in biological process (Top 10)

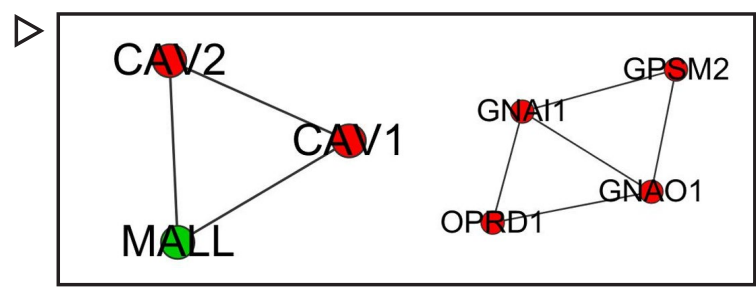

\begin{tabular}{cccl}
\hline GO & Count & Q-value & \\
\hline G0:0006355 & 111 & $8.58 \mathrm{E}-86$ & regulation of transcription, DNA-dependent \\
GO:0007165 & 119 & $1.38 \mathrm{E}-74$ & signal transduction \\
GO:0006350 & 76 & $5.92 \mathrm{E}-44$ & transcription \\
GO:0007186 & 53 & $1.10 \mathrm{E}-41$ & G-protein coupled receptor protein signaling pathway \\
GO:0007275 & 65 & $3.55 \mathrm{E}-35$ & development \\
G0:0006811 & 40 & $2.25 \mathrm{E}-33$ & ion transport \\
G0:0007608 & 26 & $1.17 \mathrm{E}-22$ & sensory perception of smell \\
GO:0055114 & 29 & $1.58 \mathrm{E}-22$ & oxidation reduction \\
G0:0006468 & 30 & $3.10 \mathrm{E}-22$ & protein amino acid phosphorylation \\
G0:0007155 & 29 & $9.12 \mathrm{E}-20$ & cell adhesion \\
\hline
\end{tabular}

for Annotation, Visualization and Integrated Discovery) software [35], respectively. Genes were enriched in biological process, cell composition and molecular function through GO enrichment analysis. Each item with gene count $>2$ and p-value $<0.01$ were chosen as the criterion.

\section{Results}

Screening of DEGs and differentially expressed miRNAs

Totally, 403 DEGs and 231 differentially expressed miRNAs were identified in RCC samples compared with normal controls with the p-value $<0.05$ and $|\log 2 \mathrm{FC}|>1$, respectively. Besides, the results of CNV analysis showed that 1369 genes and 68 miRNAs were isolated from the genome regments with CNV.

Network construction and functional modules

A total of 209 target genes related to 59 differentially expressed miRNAs that associated with RCC was selected based on the 6 miRNA prediction algorithm. Finally, 340 interaction pairs among these CNV related genes and miRNAs were predicted to construct the network (Fig. 1). As a result, a high level of connectivity is shown in the network. All these nodes were related to RCC and several nodes were highly in degrees in the network.

In addition, 2 significant modules with degree $>2$ in specific RCC regulatory network were isolated. As shown in Fig. 2, CNV related genes caveolin 1 (CAV1) and caveolin 2 (CAV2) were linked with DEG mal-T-cell differentiation protein (MALL). Notably, MALL was downregulated genes. Besides, guanine nucleotide binding protein (G protein)-alpha inhibiting activity polypeptide 1 (GNAI1), G-protein signaling modulator 2 (GPSM2), opioid receptordelta 1(OPRD1) and guanine nucleotide binding protein-alpha $\mathrm{O}$ (GNAO1) were also closely correlated.

Functional annotation of GO and pathway

The functional enrichment analysis of all the selected genes showed that several important GO terms in biological process, such as regulation of transcription and DNAdependent (GO: 0006355), G-protein coupled receptor protein signaling pathway (GO: 0007186), oxidation reduction (GO: 0055114) and cell adhesion (GO: 0007155) were enriched (Table 2). Besides, genes were also enriched in cell composition and molecular 


\section{Kidney Blood Pressure Research}

Table 3. GO functional enrichment in cell composition (Top 10)

\begin{tabular}{cccl}
\hline G0 & Count & Q-value & \multicolumn{1}{c}{ Description } \\
\hline GO:0005634 & 289 & $9.39 \mathrm{E}-254$ & nucleus \\
G0:0016021 & 256 & $3.64 \mathrm{E}-205$ & integral to membrane \\
GO:0005737 & 262 & $1.09 \mathrm{E}-182$ & cytoplasm \\
G0:0016020 & 258 & $2.96 \mathrm{E}-176$ & membrane \\
G0:0005886 & 183 & $1.37 \mathrm{E}-148$ & plasma membrane \\
G0:0005576 & 124 & $2.12 \mathrm{E}-108$ & extracellular region \\
G0:0005887 & 85 & $6.45 \mathrm{E}-81$ & integral to plasma membrane \\
G0:0005829 & 56 & $2.06 \mathrm{E}-48$ & cytosol \\
GO:0005739 & 56 & $3.16 \mathrm{E}-46$ & mitochondrion \\
G0:0005783 & 46 & $5.88 \mathrm{E}-37$ & endoplasmic reticulum \\
\hline
\end{tabular}

Table 4. GO functional enrichment in molecular function (Top 10)

\begin{tabular}{cccl}
\hline GO & Count & Q-value & \multicolumn{1}{c}{ Description } \\
\hline GO:0005515 & 351 & $6.39 \mathrm{E}-287$ & protein binding \\
GO:0008270 & 147 & $8.76 \mathrm{E}-133$ & zinc ion binding \\
GO:0000166 & 129 & $3.35 \mathrm{E}-116$ & nucleotide binding \\
GO:0005524 & 107 & $4.27 \mathrm{E}-107$ & ATP binding \\
GO:0046872 & 149 & $4.87 \mathrm{E}-100$ & metal ion binding \\
GO:0016740 & 92 & $4.18 \mathrm{E}-77$ & transferase activity \\
GO:0004872 & 85 & $1.62 \mathrm{E}-67$ & receptor activity \\
GO:0003700 & 59 & $2.01 \mathrm{E}-52$ & transcription factor activity \\
GO:0043565 & 41 & $5.07 \mathrm{E}-40$ & sequence-specific DNA binding \\
GO:0003677 & 68 & $3.09 \mathrm{E}-39$ & DNA binding \\
\hline
\end{tabular}

Table 5. Pathway enrichment in RCC (Top 10)

\begin{tabular}{lccl}
\hline \multicolumn{1}{c}{ KEGG } & Count & Q-value & \multicolumn{1}{c}{ Description } \\
\hline KEGG_PATHWAY & 25 & $4.93 \mathrm{E}-11$ & Neuroactive ligand-receptor interaction \\
KEGG_PATHWAY & 24 & $1.95 \mathrm{E}-10$ & Cytokine-cytokine receptor interaction \\
KEGG_PATHWAY & 21 & $3.97 \mathrm{E}-10$ & Focal adhesion \\
KEGG_PATHWAY & 16 & $8.21 \mathrm{E}-09$ & Tight junction \\
KEGG_PATHWAY & 22 & $8.21 \mathrm{E}-09$ & MAPK signaling pathway \\
KEGG_PATHWAY & 25 & $2.83 \mathrm{E}-08$ & Olfactory transduction \\
KEGG_PATHWAY & 15 & $4.62 \mathrm{E}-08$ & Insulin signaling pathway \\
KEGG_PATHWAY & 12 & $7.96 \mathrm{E}-08$ & ErbB signaling pathway \\
KEGG_PATHWAY & 17 & $2.80 \mathrm{E}-07$ & Regulation of actin cytoskeleton \\
KF_fr_PATHWAY & 10 & 280F-07 & Clinma
\end{tabular}

function, and the most significant GO terms were nucleus (GO:0005634) and protein binding (G0:0005515), respectively (Table 3 and 4). On the other hand, the top 10 pathways of these genes were also enriched (Table 5). The results showed that the most significant pathway was neuroactive ligand-receptor interaction. In addition, the other significant pathways such as Cytokine-cytokine receptor interaction, Focal adhesion, Tight junction, MAPK signaling pathway, Olfactory transduction, Insulin signaling pathway, ErbB signaling pathway, Regulation of actin cytoskeleton and Glioma were also enriched.

\section{Discussion}

RCC is a common cancer with complex mechanism by the combination of multiple genes. In this study, we utilized comprehensive bioinformatics methods to explore the potential molecular mechanism of RCC. The DEGs, differentially expressed miRNAs, CNV related 


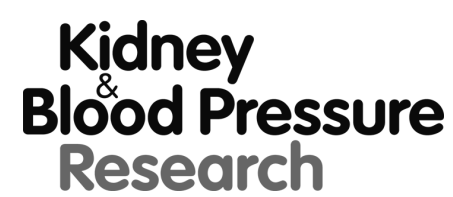

Kidney Blood Press Res 2015;40:288-297

\begin{tabular}{l|l}
\hline DOI: $10.1159 / 000368504$ & C 2015 S. Karger AG, Basel
\end{tabular}

Published online: May 27, 2015

www.karger.com/kbr

genes and miRNAs, and target genes for miRNAs that associated with RCC were analyzed based on three microarrays. Moreover, we selected 2 significnat modules with several key DEGs (like CAV1, CAV2, GNAI1, GPSM2 and GNAO1) in RCC regulatory network. Besides, functional enrichment analyses showed that some of the GO terms and pathways, such as signal transduction, G-protein coupled receptor protein signaling pathway, focal adhesion, MAPK signaling pathway and neuroactive ligand-receptor interaction, were also involved with the regulation of RCC.

Our data showed that several key genes had close relationships with RCC from the 2 significant modules. CAV1 is a tumor suppressor candidate and is a negative regulator of the Ras-p42/44 mitogen-activated kinase cascade. Loss of CAV1 in the tumour microenvironment contributes to the metastatic behaviour of tumour cells by up-regulation of TGF- $\beta 1$ and SNCG through Akt activation [36]. Its expression is down-regulated in some tumors, however, many studies demonstrates that elevated expression of CAV1 is associated with tumor metastasis and a worse prognosis in several neoplasms, such as colon and urinary bladder cancer [37, 38]. In the kidney, one study verified that overexpression of CAV1 in RCC correlated with a poor prognosis [39]. Holger et al. proved that abbrevant expression of CAV1 was related to the metastasis formation and cytogenetic abnormalities of kidney cancer [40]. Also, Zucchini et al. demonstrated that up-regulation of CAV1 that involved with cell-cell adhesion and cell growth was observed in kidney cancer cells [41]. Besides, CAV1 can be used in differentiating chromophobe RCC from oncocytoma and the effects are superior to CK7 [42]. Therefore, we speculate that CAV1 overexpression may contribute to RCC progression and correlate with a poor prognosis. Meanwhile, CAV2 belongs to the same family of CAV1, which also functions as a tumor suppressor. A silencing study of CAV2 revealed significant inhibition of cell proliferation, migration and invasion through dysregulation of the focal adhesion pathway, and its expression was significantly up-regulated in RCC clinical specimens [43].

On the other hand, GNAI1, GPSM2 and GNAO1 were members of the G protein signal transduction family. G-protein coupled receptor protein signaling pathway is frequently associated with tumorigenesis. It has been reported that there were $4.2 \%$ of tumours carry activating mutations in GNAS and nearly $20 \%$ of human tumours harbour mutations in G proteins and G-protein-coupled receptors (GPCRs). Besides, many human cancer-associated viruses also express constitutively active viral GPCRs [44]. As previous reported, GNAI1 can suppresses tumor cell migration and invasion [45], GPSM2 may influence the cell division in cancer [46], and GNAO1 is demonstrated to play an important role in oncogenesis [47]. Combination with these findings, we speculate that G-protein coupled receptor protein signaling pathway may play an important role in RCC initiation, and these genes are suspected to participate in the process of RCC through involved in this pathway.

Besides, RCC is shown to produce particular extracellular matrix components and expresses a characteristic repertoire of cell adhesion molecules. The expression of the alpha nu beta 3 integrin subunit is differentially expressed in all types of renal cell tumors [48]. Zucchini et al. demonstrated that up-regulation of CAV1 was involved with cell-cell adhesion in kidney cancer cells [41]. Therefore, focal adhesion factors are also certified to participate in the process of RCC. In polycystic kidney disease (PKD), MAPK signaling pathway and neuroactive ligand receptor interaction were enriched. The expression of genes involved in MAPK pathway is significantly higher in PKD, indicating the activation of MAPK signalling pathway [49]. Based on our results, we speculate that changes in the former pathways may cause tissue lesions and even lead to the generation of RCC.

No experimental validation is a limitation of our study, further experimental studies are still needed. Moreover, there are some sub-types of cancers with distinct phenotypes and control samples in the three datasets in this study, separate sub-analyses of the different sub-types of cancers versus the controls should be considered to validate these genes and pathways. 


\section{Kidney \\ Blood Pressure Research}

Liu/Wang/Sun: Isolate Genes and Pathways in Renal Cell Carcinoma

\section{Conclusion}

To sum up, CAV1 may contibute to the progerssion of RCC and correlate with a poor prognosis. Loss of CAV2 may reveal significant inhibition of cell proliferation, migration and invasion. GNAI1, GPSM2 and GNAO1 are likely involved in the regulation of RCC through G protein signal transduction. Besides, G-protein coupled receptor protein signaling pathway, focal adhesion, MAPK signaling pathway and neuroactive ligand receptor interaction are enriched in the development of RCC and may result in the development of this disease. Our study may provide theirotical basis for the future study of RCC diagnosis or treatment.

\section{Disclosure Statement}

The authors declare that they have no conflict of interest.

\section{Acknowledgements}

We received no funding for this study.

\section{Reference}

Chow WH, Devesa SS, Warren JL, Fraumeni JF, Jr: Rising incidence of renal cell cancer in the United States. JAMA 1999;281:1628-1631.

2 Cohen HT, McGovern FJ: Renal-cell carcinoma. N Engl J Med 2005;353:2477-2490.

3 Chow W-H, Dong LM, Devesa SS: Epidemiology and risk factors for kidney cancer. Nature Rev Urol 2010;7:245-257.

4 Finley DS, Pantuck AJ, Belldegrun AS: Tumor biology and prognostic factors in renal cell carcinoma. Oncologist 2011;16 Suppl 2:4-13.

5 Datta K, Sundberg C, Karumanchi SA, Mukhopadhyay D: The 104-123 amino acid sequence of the $\beta$-domain of von Hippel-Lindau gene product is sufficient to inhibit renal tumor growth and invasion. Cancer Res 2001;61:1768-1775.

6 Angevin E, Lopez-Martin JA, Lin C-C, Gschwend JE, Harzstark A, Castellano D, Soria J-C, Sen P, Chang J, Shi M: Phase I study of dovitinib (TKI258), an oral FGFR, VEGFR, and PDGFR inhibitor, in advanced or metastatic renal cell carcinoma. Clin Cancer Res 2013;19:1257-1268.

7 Figlin RA: From the guest editor: renal cell carcinoma the next decade of development. Cancer J (Sudbury, Mass) 2013;19:297.

8 Wiesener MS, Münchenhagen PM, Berger I, Morgan NV, Roigas J, Schwiertz A, Jürgensen JS, Gruber G, Maxwell PH, Löning SA: Constitutive activation of hypoxia-inducible genes related to overexpression of hypoxia-inducible factor- $1 \alpha$ in clear cell renal carcinomas. Cancer Res 2001;61:5215-5222.

9 Costa LJ, Drabkin HA: Renal cell carcinoma: new developments in molecular biology and potential for targeted therapies. Oncologist 2007;12:1404-1415.

10 Pelletier J, Dayan F, Durivault J, Ilc K, Pecou E, Pouyssegur J, Mazure N: The asparaginyl hydroxylase factor-inhibiting HIF is essential for tumor growth through suppression of the p53-p21 axis. Oncogene 2012;31:2989-3001.

11 Ku BM, Kim D-S, Kim K-H, Yoo BC, Kim S-H, Gong Y-D, Kim S-Y: Transglutaminase 2 inhibition found to induce p53 mediated apoptosis in renal cell carcinoma. FASEB J 2013;27:3487-3495.

12 Li X, Xin S, He Z, Che X, Wang J, Xiao X, Chen J, Song X: MicroRNA-21 (miR-21) Post-Transcriptionally Downregulates Tumor Suppressor PDCD4 and Promotes Cell Transformation, Proliferation, and Metastasis in Renal Cell Carcinoma. Cell Physiol Biochem 2014;33:1631-1642.

13 Ivy SP, Wick JY, Kaufman BM: An overview of small-molecule inhibitors of VEGFR signaling. Nat Rev Clin Oncol 2009;6:569-579. 


\section{Kidney \\ Blood Pressure Research}

Liu/Wang/Sun: Isolate Genes and Pathways in Renal Cell Carcinoma

14 Ferrara N, Hillan KJ, Novotny W: Bevacizumab (Avastin), a humanized anti-VEGF monoclonal antibody for cancer therapy. Biochem Biophys Res Commun 2005;333:328-335.

15 Del Bufalo D, Ciuffreda L, Trisciuoglio D, Desideri M, Cognetti F, Zupi G, Milella M: Antiangiogenic potential of the mammalian target of rapamycin inhibitor temsirolimus. Cancer Res 2006;66:5549-5554.

16 Li W, Zhu W, Che J, Sun W, Liu M, Peng B, Zheng J: Microarray profiling of human renal cell carcinoma: identification for potential biomarkers and critical pathways. Kidney Blood Press Res 2012;37:506-513.

17 Gan B, Lim C, Chu G, Hua S, Ding Z, Collins M, Hu J, Jiang S, Fletcher-Sananikone E, Zhuang L: FoxOs enforce a progression checkpoint to constrain mTORC1-activated renal tumorigenesis. Cancer Cell 2010;18:472484.

18 Zaravinos A, Lambrou GI, Mourmouras N, Katafygiotis P, Papagregoriou G, Giannikou K, Delakas D, Deltas C: New miRNA Profiles Accurately Distinguish Renal Cell Carcinomas and Upper Tract Urothelial Carcinomas from the Normal Kidney. PloS One 2014;9:e91646.

19 Krill-Burger JM, Lyons MA, Kelly LA, Sciulli CM, Petrosko P, Chandran UR, Kubal MD, Bastacky SI, Parwani AV, Dhir R: Renal Cell Neoplasms Contain Shared Tumor Type-Specific Copy Number Variations. Am J Pathol 2012;180:2427-2439.

20 Team TRDC. R: A language and environment for statistical computing. R Foundation Statistical Computing, Vienna, Austria, 2008.

21 Irizarry RA, Hobbs B, Collin F, Beazer-Barclay YD, Antonellis KJ, Scherf U, Speed TP: Exploration, normalization, and summaries of high density oligonucleotide array probe level data. Biostatistics 2003;4:249-264.

22 Mills RE, Pittard WS, Mullaney JM, Farooq U, Creasy TH, Mahurkar AA, Kemeza DM, Strassler DS, Ponting $\mathrm{CP}$, Webber C: Natural genetic variation caused by small insertions and deletions in the human genome. Genome Res 2011;21:830-839.

23 Smyth GK: Limma: linear models for microarray data, In: Bioinf Computat Biol Solut Using R Bioconduct. Springer Verlag, 2005, pp. 397-420.

24 Wang K, Li M, Hadley D, Liu R, Glessner J, Grant SF, Hakonarson H, Bucan M: PennCNV: an integrated hidden Markov model designed for high-resolution copy number variation detection in whole-genome SNP genotyping data. Genome Res 2007;17:1665-1674.

25 Kwong S, Chau C, Man K-F, Tang K-S: Optimisation of HMM topology and its model parameters by genetic algorithms. Pattern Rec 2001;34:509-522.

26 Krek A, Grun D, Poy MN, Wolf R, Rosenberg L, Epstein EJ, MacMenamin P, da Piedade I, Gunsalus KC, Stoffel M, Rajewsky N: Combinatorial microRNA target predictions. Nat Genet 2005;37:495-500.

27 Kiriakidou M, Nelson PT, Kouranov A, Fitziev P, Bouyioukos C, Mourelatos Z, Hatzigeorgiou A: A combined computational-experimental approach predicts human microRNA targets. Genes Dev 2004;18:1165-1178.

28 John B, Enright AJ, Aravin A, Tuschl T, Sander C, Marks DS: Human MicroRNA targets. PLoS Biol 2004;2:e363.

29 Rehmsmeier M, Steffen P, Hochsmann M, Giegerich R: Fast and effective prediction of microRNA/target duplexes. RNA 2004;10:1507-1517.

30 Miranda KC, Huynh T, Tay Y, Ang YS, Tam WL, Thomson AM, Lim B, Rigoutsos I: A pattern-based method for the identification of MicroRNA binding sites and their corresponding heteroduplexes. Cell 2006;126:12031217.

31 Lewis BP, Shih IH, Jones-Rhoades MW, Bartel DP, Burge CB: Prediction of mammalian microRNA targets. Cell 2003;115:787-798.

32 Smoot ME, Ono K, Ruscheinski J, Wang P-L, Ideker T: Cytoscape 2.8: new features for data integration and network visualization. Bioinformatics 2011;27:431-432.

33 Consortium GO: Gene Ontology annotations and resources. Nucleic Acids Res 2013;41:D530-D535.

34 Kanehisa M, Goto S, Kawashima S, Okuno Y, Hattori M: The KEGG resource for deciphering the genome. Nucleic Acids Res 2004;32:D277-D280.

35 Dennis G Jr, Sherman BT, Hosack DA, Yang J, Gao W, Lane HC, Lempicki RA: DAVID: database for annotation, visualization, and integrated discovery. Genome Biol 2003;4:P3.

36 Ayala G, Morello M, Frolov A, You S, Li R, Rosati F, Bartolucci G, Danza G, Adam RM, Thompson TC, Lisanti MP, Freeman MR, Di Vizio D: Loss of Caveolin-1 in Prostate Cancer Stroma Correlates with Reduced Relapse-Free Survival and is Functionally Relevant to Tumor Progression. J Pathol 2013; 231:77-87. 


\section{Kidney \\ Blood Pressure Research}

37 Fine SW, Lisanti MP, Galbiati F, Li M: Elevated expression of caveolin-1 in adenocarcinoma of the colon. Am J Clin Pathol 2001;115:719-724.

38 Rajjayabun P, Garg S, Durkan G, Charlton R, Robinson M, Mellon J: Caveolin-1 expression is associated with high-grade bladder cancer. Urology 2001;58:811-814.

39 Campbell L, Gumbleton M, Griffiths DFR: Caveolin-1 overexpression predicts poor disease-free survival of patients with clinically confined renal cell carcinoma. Brit J Cancer 2003;89:1909-1913.

40 Sültmann H, von Heydebreck A, Huber W, Kuner R, Buneß A, Vogt M, Gunawan B, Vingron M, Füzesí L, Poustka A: Gene expression in kidney cancer is associated with cytogenetic abnormalities, metastasis formation, and patient survival. Clin Cancer Res 2005;11:646-655.

41 Zucchini C, Bianchini M, Valvassori L, Perdichizzi S, Benini S, Manara MC, Solmi R, Strippoli P, Picci P, Carinci P, Scotlandi K: Identification of candidate genes involved in the reversal of malignant phenotype of osteosarcoma cells transfected with the liver/bone/kidney alkaline phosphatase gene. Bone 2004;34:672679.

42 Garcia E, Li M: Caveolin-1 immunohistochemical analysis in differentiating chromophobe renal cell carcinoma from renal oncocytoma. Am J Clin Pathol 2006;125:392-398.

43 Yamasaki T, Seki N, Yoshino H, Itesako T, Hidaka H, Yamada Y, Tatarano S, Yonezawa T, Kinoshita T, Nakagawa M, Enokida H: MicroRNA-218 Inhibits Cell Migration and Invasion in Renal Cell Carcinoma through Targeting Caveolin-2 Involved in Focal Adhesion Pathway. J Urol 2013;190:1059-1068.

44 O'Hayre M, Vazquez-Prado J, Kufareva I, Stawiski EW, Handel TM, Seshagiri S, Gutkind JS: The emerging mutational landscape of G proteins and G-protein-coupled receptors in cancer. Nat Rev Cancer 2013;13:412-424.

45 Jin Y, Chen D, Cabay RJ, Wang A, Crowe DL, Zhou X: Role of microRNA-138 as a Potential Tumor Suppressor in Head and Neck Squamous Cell Carcinoma. Int Rev Cell Molec Biol 2013;303:357.

46 Fukukawa C, Ueda K, Nishidate T, Katagiri T, Nakamura Y: Critical roles of LGN/GPSM2 phosphorylation by PBK/TOPK in cell division of breast cancer cells. Genes, Chromosomes Cancer 2010;49:861-872.

47 Kan Z, Jaiswal BS, Stinson J, Janakiraman V, Bhatt D, Stern HM, Yue P, Haverty PM, Bourgon R, Zheng J: Diverse somatic mutation patterns and pathway alterations in human cancers. Nature 2010;466:869-873.

48 Droz D, Patey N, Paraf F, Chretien Y, Gogusev J: Composition of extracellular matrix and distribution of cell adhesion molecules in renal cell tumors. Lab Invest 1994;71:710-718.

49 Pandey P, Brors B, Srivastava PK, Bott A, Boehn SN, Groene HJ, Gretz N: Microarray-based approach identifies microRNAs and their target functional patterns in polycystic kidney disease. BMC Genomics 2008;9:624. 